\title{
Quantum Mechanics and the Philosophy of Language: Reconsideration of Traditional Philosophies
}

\author{
Shiro Ishikawa \\ Department of Mathematics, Faculty of Science and Technology, Keio University, Yokohama, Japan \\ Email: ishikawa@math.keio.ac.jp
}

Received November 10, 2011; revised December 15, 2011; accepted December 24, 2011

\begin{abstract}
Recently we proposed "a new interpretation of quantum mechanics (called quantum and classical measurement theory)" in this journal (JQIS: Vol. 1, No. 2), which was characterized as the metaphysical and linguistic turn of quantum mechanics. This turn from physics to language does not only realize the remarkable extension of quantum mechanics but also yield the quantum mechanical world view (i.e., the philosophy of quantum mechanics). And thus, the turn urges us to dream that traditional philosophies (i.e., Parmenides, Plato, Aristotle, Descartes, John Locke, Berkeley, Hume, Kant, Saussure, Wittgenstein, etc.) can be understood in the quantum mechanical world view. This dream will be challenged in this paper. We, of course, know that most scientists are skeptical to philosophy. Still, we can expect that readers find a good linguistic philosophy (i.e. philosophy of language) in quantum mechanics.
\end{abstract}

Keywords: Copenhagen Interpretation; Operator Algebra; Language Game; Mind-Body Problem; Space-Time Problem; Leibniz-Clarke Correspondence; McTaggart's Paradox; Schrödinger's Cat; Zeno’s Paradoxes; Syllogism; EPR-Paradox

\section{Introduction}

Recently in [1-6] (finally, in [1]), we proposed (classical and quantum) measurement theory, which is characterized as the linguistic (or, metaphysical) approach to quantum mechanics. As seen in [1-6], this theory includes several conventional system theories (e.g. quantum system theory, statistics, dynamical system theory and so on). And thus, we believe that measurement theory is one of the most applicable theories in science.

The purpose of this paper is to show that quantum mechanics has a good linguistic philosophy, that is, to understand traditional philosophies in the framework of measurement theory, or equivalently, to explain the meaning of Figure 1. As shown in this paper, we consider that Figure 1 is natural, since the purposes of all theories in this figure are the same, that is, the world description (i.e., how to describe the world).

\section{Measurement Theory}

In this section, according to [1], we explain the outline (Figure 1: (2)-(3)) of measurement theory (or in short, MT).

\subsection{Axioms 1 and 2 of MT (Figure 1: (2)-(3)}

Measurement theory ([1-6]) is, by an analogy of quantum mechanics (or, as a linguistic turn of quantum mechanics, $c f$. Figure 1: (3)), constructed as the mathematical theory formulated in a certain $C^{*}$-algebra $A$ (i.e., a norm closed sub algebra in $B(H), c f .[7,8])$. MT is composed of two axioms as follows:

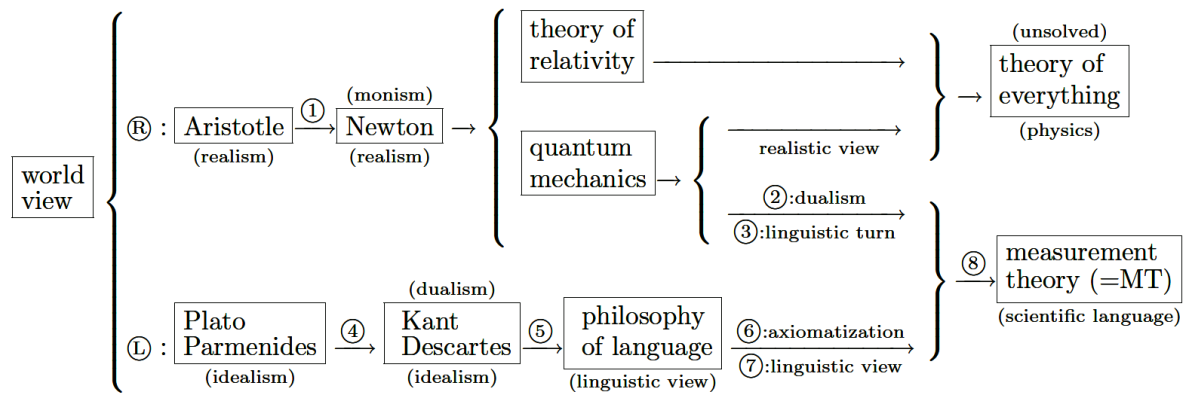

Figure 1. This figure (1)-8) will be explained in this paper. 


$$
(A) \underset{(\text { language })}{[\mathrm{MT}]}=[\text { measurement }]+[\text { causality }]
$$

For completeness, note that measurement theory $(A)$ is not physics but a kind of language based on "the quantum mechanical world view".

When $A=B_{c}(H)$, the $C^{*}$-algebra composed of all compact operators on a Hilbert space $H$, the $(A)$ is called quantum measurement theory (or, quantum system theory), which can be regarded as the linguistic aspect of quantum mechanics. Also, when $A$ is commutative (that is, when $A$ is characterized by $C_{0}(\Omega)$, the $C^{*}$-algebra composed of all continuous complex-valued functions vanishing at infinity on a locally compact Hausdorff space $\Omega(c f$. [7])), the $(A)$ is called classical measurement theory. Thus, we have the following classification:

$$
\text { (B) MT }\left\{\begin{array}{l}
\text { quantum MT }\left(\text { when } A=B_{c}(H)\right) \\
\text { classical MT }\left(\text { when } A=C_{0}(\Omega)\right)
\end{array}\right.
$$

That is, this theory covers several conventional system theories (i.e., statistics, dynamical system theory, quantum system theory, etc.).

Now we shall explain the measurement theory $(A)$. Let $A(\subseteq B(H))$ be a $C^{*}$-algebra, and let $A^{*}$ be the dual Banach space of $A$. The norm $\|\rho\|_{A^{*}}$ is defined by sup $\left\{\|\rho(F)\| F \in A\right.$ such that $\left.\|F\|_{A}\left(=\|F\|_{B(H)}\right) \leq 1\right\}$.

Define the mixed state $\rho\left(\in A^{*}\right)$ such that $\|\rho\|_{A^{*}}=1$ and $\rho(F) \geq 0$ for all $F \in A$ such that $F \geq 0$. And put

$$
S^{m}\left(A^{*}\right)=\left\{\rho \in A^{*} \mid \rho \text { is a mixed state }\right\} \text {. }
$$

A mixed state $\rho$ is called a pure state if it satisfies that " $\rho=\theta \rho_{1}+(1-\theta) \rho_{2}$ for some $\rho_{1}, \rho_{2} \in S^{m}\left(A^{*}\right)$ and $0<$ $\theta<1$ " implies $\rho=\rho_{1}=\rho_{2}$. Put

$$
S^{p}\left(A^{*}\right)=\left\{\rho \in S^{m}\left(A^{*}\right) \mid \rho \text { is a pure state }\right\},
$$

which is called a state space. It is well known (cf. [7]) that

$S^{p}\left(B_{c}(H)^{*}\right)=\left\{|u\rangle\langle u|(i . e .\right.$, the Dirac notation $\left.)\|u\|_{H}=1\right\}$, and $S^{p}\left(C_{0}(\Omega)^{*}\right)=\left\{\delta_{\omega_{0}} \mid \delta_{\omega_{0}}\right.$ is a point measure at $\left.\omega_{0} \in \Omega\right\}$, where $\int_{\Omega} f(\omega) \delta_{\omega_{0}}(\mathrm{~d} \omega)=f\left(\omega_{0}\right)\left(\forall f \in C_{0}(\Omega)\right)$. The latter implies that $S^{p}\left(C_{0}(\Omega)^{*}\right)$ can be also identified with $\Omega$ (called a spectrum space, spectrum or maximal ideal space) such as

$$
S^{p}\left(C_{0}(\Omega)^{*}\right) \ni \delta_{\omega} \leftrightarrow \omega \in \Omega
$$

In this paper we assume that the $C^{*}$-algebra $A(\subseteq B(H))$ has the identity $I$. This assumption is not unnatural, since, if $I \notin A$, it suffices to reconstruct the above $A$ such that it includes $A \cup\{I\}$.

According to the noted idea ( $c f$. [9]) in quantum mechanics, an observable $O:=\left(X, B_{X}, F\right)$ in $A$ is defined as follows:

[1): Field] $X$ is a set, $B_{X}\left(\subseteq 2^{X}\right.$, the power set of $\left.X\right)$ is a field of $X$, that is, " $\Xi_{1}, \Xi_{2} \in B_{X} \Rightarrow \Xi_{1} \cup \Xi_{2} \in B_{X}$ ", " $\Xi \in B_{X} \Rightarrow X \quad \Xi \in B_{X}$ ".

[2): Additivity] $F$ is a mapping from $B_{X}$ to $A$ satisfying: 1): for every $\Xi \in B_{X}, F(\Xi)$ is a non-negative element in $A$ such that $0 \leq F(\Xi) \leq I ; 2): F(\varnothing)=0$ and $F(X)=I$, where 0 and $I$ is the 0 -element and the identity in $A$ respectively; 3 ): for any $\Xi_{1}, \Xi_{2} \in B_{X}$ such that $\Xi_{1} \cap \Xi_{2}=\varnothing$, it holds that $F\left(\Xi_{1} \cup \Xi_{2}\right)=F\left(\Xi_{1}\right)+$ $F\left(\Xi_{2}\right)$.

As the simplest observable, the existence observable $O^{(e)}=\left(X,\{\varnothing, X\}, F^{(e)}\right)$ is defined such that $F^{(e)}(\varnothing)=$ 0 and $F^{(e)}(X)=I$.

With anysystem $S$, a $C^{*}$-algebra $A(\subseteq B(H))$ can be associated in which the measurement theory $(A)$ of that system can be formulated. A state of the system $S$ is represented by an element $\rho\left(\in S^{p}\left(A^{*}\right)\right)$, and an observable is represented by an observable $O:=\left(X, B_{X}, F\right)$ in $A$. Also, the measurement of the observable $O$ for the system $S$ with the state $\rho$ is denoted by $M_{A}\left(O, S_{[\rho]}\right)$ (or more precisely, $\left.M_{A}\left(O:=\left(X, B_{X}, F\right), S_{[\rho]}\right)\right)$. An observer can obtain a measured value $x(\in X)$ by the measurement $M_{A}\left(O, S_{[\rho]}\right)$.

The axiom presented below is a kind of mathematical generalization of Born's probabilistic interpretation of quantum mechanics. And thus, it is a statement without reality.

Axiom 1. [Measurement]. The probability that a measured value $x(\in X)$ obtained by the measurement $M_{A}\left(O:=\left(X, B_{X}, F\right), S_{\left(\rho_{0}\right)}\right)$ belongs to a set $\Xi\left(\in B_{X}\right)$ is given by $\rho_{0}(F(\Xi))$.

Next, we explain Axiom 2 in $(A)$. Let $(T, \leq)$ be a tree, i.e., a partial ordered finite set such that " $t_{1} \leq t_{3}$ and $t_{2} \leq$ $t_{3}$ " implies " $t_{1} \leq t_{2}$ or $t_{2} \leq t_{1}$ ". Assume that there exists an element $t_{0} \in T$, called the root of $T$, such that $t_{0} \leq t$ $(\forall t \in T)$ holds. Put $T_{\leq}^{2}=\left\{\left(t_{1}, t_{2}\right) \in T^{2} \mid t_{1} \leq t_{2}\right\}$. The family $\left\{\Phi_{t_{1}, t_{2}}: A_{t_{2}} \rightarrow A_{t_{1}}\right\}_{\left(t_{1}, t_{2}\right) \in T_{\leq}^{2}}$ is called a Markov relation (due to the Heisenberg picture), if it satisfies the following conditions 1) and 2).

1): With each $t \in T$, a $C^{*}$-algebra $A_{t}$ is associated.

2): For each $\left(t_{1}, t_{2}\right) \in T_{\leq}^{2}$, a Markov operator $\Phi_{t_{1}, t_{2}}: A_{t_{2}} \rightarrow A_{t_{1}}$ is defined. And it satisfies that $\Phi_{t_{1}, t_{2}} \Phi_{t_{2}, t_{3}}=\Phi_{t_{1}, t_{3}}$ holds for any $\left(t_{1}, t_{2}\right),\left(t_{2}, t_{3}\right) \in T_{\leq}^{2}$. 
The family of dual operators

$\left\{\Phi_{t_{1}, t_{2}}^{*}: S^{m}\left(A_{t_{1}}^{*}\right) \rightarrow S^{m}\left(A_{t_{2}}^{*}\right)\right\}_{\left(t_{1}, t_{2}\right) \in T_{\Sigma}^{2}}$ is called a dual

Markov relation (due to the Schrödinger picture). However, it is not formally used in measurement theory.

Now Axiom 2 in the measurement theory $(A)$ is presented as follows:

Axiom 2. [Causality]. The causality is represented by a Markov relation $\left\{\Phi_{t_{1}, t_{2}}: A_{t_{2}} \rightarrow A_{t_{1}}\right\}_{\left(t_{1}, t_{2}\right) \in T_{\leq}^{2}}$.

For the further argument (i.e., the $W^{*}$-algebraic formulation) of measurement theory, see Appendix in [1].

\subsection{Linguistic Interpretation (Figure 1: (2), (3))}

The measurement theory $(A)$ asserts

(C): Obey Axioms 1 and 2. And, describe any ordinary phenomenon according to Axioms 1 and 2 (in spite that Axioms 1 and 2 can not be tested experimentally).

Still, most readers may be perplexed how to use Axioms 1 and 2 since there are various usages. Thus, the following problem is significant.

(D): How should Axioms 1 and 2 be used?

Note that reality is not reliable since Axioms 1 and 2 are statements without reality.

Here, in spite of the linguistic turn (Figure 1: (3) and the mathematical generalization from $B(H)$ to a $C^{*}$ algebra $A$, we consider that the dualism (i.e. the spirit of so called Copenhagen interpretation) of quantum mechanics is inherited to measurement theory (Figure 1: (2)). Thus, we present the following interpretation $(E)$ $\left[=\left(E_{1}\right)-\left(E_{3}\right)\right]$. That is, as the answer to the question $(D)$, we propose:

$\left(E_{1}\right)$ Consider the dualism composed of "observer" and "system (=measuring object)". And therefore, "observer" and "system" must be absolutely separated.

$\left(E_{2}\right)$ Only one measurement is permitted. And thus, the state after a measurement is meaningless since it can not be measured any longer. Also, the causality should be assumed only in the side of system, however, a state never moves. Thus, the Heisenberg picture should be adopted.

$\left(E_{3}\right)$ Also, the observer does not have the space-time. Thus, the question: "When and where is a measured value obtained?" is out of measurement theory, and so on.

Although N. Bohr (the chief proponent of the Copenhagen interpretation) said, in the Bohr-Einstein debates $[10,11]$, that the interpretation of a physical theory has to rely on an experimental practice. However, we consider that all confusion is due to the preconception that the Copenhagen interpretation is within physics. In this sense, we agree with A. Einstein, who never accepted the Copenhagen interpretation as physics. That is, in spite of Bohr's realistic view, as mentioned in [1], we propose the following linguistic world view (Figure 1: (3)):

$(F)$ In the beginning was the language called measure- ment theory (with the interpretation $(E)$ ). And, for example, quantum mechanics can be fortunately described in this language. And moreover, almost all scientists have already mastered this language partially and informally since statistics (at least, its basic part) is characterized as one of aspects of measurement theory (cf. [1-6]).

In this sense, we consider that measurement theory holds as a kind of language-game (with the rule (Axioms 1 and 2, Interpretation $(E)$ ), and therefore, measurement theory is regarded as the axiomatization (Figure 1: 6) of the philosophy of language (i.e. Saussure's linguistic world view).

\section{The Quantum Mechanical World View}

In this main section, we shall explain Figure 1: (1)-8) from the measurement theoretical point of view.

\subsection{Realistic World View and Linguistic World View}

Figure 1 says that the realistic world view $\AA$ and the linguistic world view (D) exist together in science. Some may ask:

$(G)$ : Why is the series (D) (or, idealism originated by Plato) underestimated in science?

We think that the reason is due to the fact that the (D) is lacking in the axiomatization (6) if we do not have measurement theory. That is, we believe that there is no scientific world view without axiomatization.

\subsection{Dualism}

Interpretation $\left(E_{1}\right)$ says "Image Figure 2 whenever measurement theory is used". where the interaction [aand (b)] must not be emphasized, that is, it must be implicit. That is because, if it is explicitly stated, the dualism $\left(E_{1}\right)$ is violated.

John Locke's famous sayings "primary quality (e.g., length, weight, etc.)" and "secondary quality (e.g., sweet, dark, cold, etc.)" urge us to associate the following correspondence:

$$
\left\{\begin{array}{l}
\text { state } \leftrightarrow \text { primary quality } \\
\text { observable } \leftrightarrow \text { secondary quality }
\end{array}\right.
$$

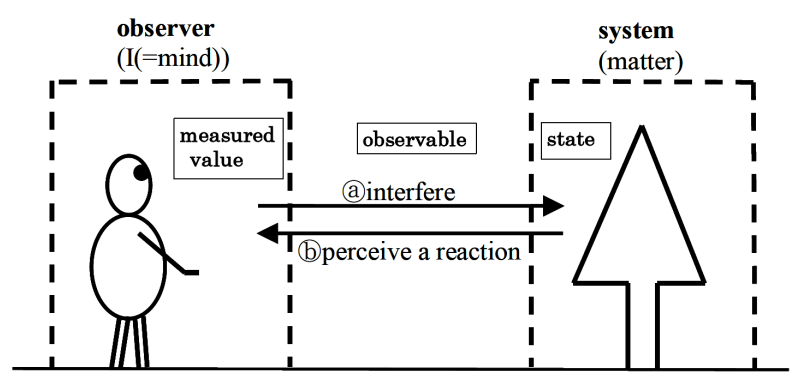

Figure 2. Descartes' figure in MT. 
Also, it may be understandable to regard "observable" as "measurement instrument" or "sensory system", for example, eyes, glasses, condensation trail (for a plane), etc. And further, it is natural to consider that there is no measured value without observer's brain (i.e., when something reaches observer's brain, it becomes a measured value). Thus, we want to consider the following correspondence in Table 1.

In the history of philosophy, two kinds of dualisms (based on "mind-body dualism" and "matter-mind dualism") may be frequently discussed. However, it should be noted that the dualism $\left(E_{1}\right)$ is composed of three concepts as mentioned in Table 1. Also, the following question is nonsense in the linguistic world view.

(H): What is "measured value" (observable, or state)? Or equivalently, in the sense of Table 1, what is "mind" (body, or matter)? And moreover, what is "probability" (or causality)?

Therefore, we must admit that the correspondence in Table 1 is rather figurative, that is, it is not worth discussing the problem $(H)$ seriously in the linguistic world view. From the great history of philosophy, we learned that the serious consideration (i.e., the consideration from the realistic world view) of the problem $(H)$ (e.g., mindbody problem, etc.) always led us into blind alleys. We have to confirm that we are now in the side of the linguistic world view (i.e., after the linguistic turn (3) in Figure 1) and not the realistic world view. Thus, our interest always focuses on the problem:

(I): How should the term: "measured value" (observable, or state) be used? And moreover, how should the term: "probability" (or causality) beused?

We, of course, assert that this answer is just given by measurement theory (i.e., Axioms 1 and 2, Interpretation (E)). After accepting measurement theory, what we can do in measurement theory is only to trust in man's linguistic competence. This is our linguistic world view $(F)$. Here, we want to consider that the following two are essentially the same:

( $\left.J_{1}\right)$ To be is to be perceived (by Berkeley).

$\left(J_{2}\right)$ There is no science without measurement (particularly, measured value).

Also, in the sense of Table 1, these are similar to $\left(J_{3}\right)$ : There is no science without human's brain, which may be also similar to Kant's assertion (see Section 3.7 later). Also, in measurement theory, the $\left(J_{1}\right)$ may say that $M_{A}\left(O, S_{[\rho]}\right)=M_{A}\left(O \times O^{(e)}, S_{[\rho]}\right)$.

Table 1. Descartes vs MT.

\begin{tabular}{cccc}
\hline Descartes & Mind (brain) & Body & Matter \\
\hline MT & Measured value & Observable & State \\
\hline
\end{tabular}

\subsection{Think, Therefore I Am}

Figure 2 (Descartes' figure in MT) may be inspired from the Descartes primary principle:

$(K)$ : I think, therefore I am.

However, it should be noted that this $(K)$ is not a statement in the dualism of Interpretation $\left(E_{1}\right)$. That is because it is natural to assume that " $\Gamma$ " = "observer" and " $P$ " = "system" in the statement $(K)$, which clearly contradicts the $\left(E_{1}\right)$. Thus, we can never expect that the $(K)$ is directly applicable in science. We may see an irony in the fact that the non-dualistic statement $(K)$ gives foundations to the dualistic Figure 2. However, it is sure that the establishment of " $I$ " in $(K)$ brought us modern science (Figure 1: (4)).

Also, it is natural to consider that Heidegger's saying: "In-der-Welt-sein" is out of Figure 2, and thus, out of measure theory. If some succeed the axiomatization of "In-der-Welt-sein", it will be the powerful rival of measurement theory in science.

\subsection{Causality and Probability}

The paradigm shift (1) in Figure 1 from "purpose (due to Aristotle)" to "causality (due to Bacon, Newton, Hume, etc.) is the greatest paradigm shift throughout all history of science. However, it should be noted that there are several ideas for "causality". For example Newton's causality is realistic, and Hume's causality is subjective. On the other hand, our causality (i.e., Axiom 2) islinguistic.

Although some philosophers (e.g., K. Popper [12]) consider that the discovery of "probability" is as great as that of "causality", it is sure that the former is underestimated in science, The reason of the underestimation may be due to the fact that the "probability" is never presented in a certain world view (but in mathematics ( $c f$. [13]), on the other hand, the "causality" is established in the world view (i.e., Newtonian mechanics). We think that it is desirable to understand the two concepts (i.e., "probability" and "causality") in the same world view. It should be noted that this is realized in Axioms 1 and 2 of measurement theory.

\subsection{Leibniz-Clarke Correspondence (Space-TimeProblem)}

In this section, first we prepare the term "spectrum space" in the Formula (3). Consider the pair $[A, \bar{A}]_{B(H)}$, called a basic structure (cf. Appendix in [1]). Here, $A(\subseteq B(H))$ is a $C^{*}$-algebra, and $\bar{A}$ ( $\left.A \subseteq \bar{A} \subseteq B(H)\right)$ is a particular $C^{*}$-algebra (called a $W^{*}$-algebra) such that $\bar{A}$ is the weak closure of $A$ in $B(H)$. Let $A_{S}(\subseteq \bar{A})$ be the commutative $C^{*}$-subalgebra, which is represented by $C_{0}\left(\Omega_{S}\right)$ for some locally compact Hausdorff space $\Omega_{S} \quad(c f$. [7]). The 
$\Omega_{S}$ iscalled a spectrum space. For example, consider one particle quantum system, formulated in a basic structure $\left[B_{c}\left(L^{2}\left({ }^{3}\right)\right), B\left(L^{2}\left({ }^{3}\right)\right)\right]_{B\left(L^{2}\left({ }^{3}\right)\right)}$. Then, we can choose the commutative $C^{*}$-algebra $C_{0}\left({ }^{3}\right)\left(\subset B\left(L^{2}\left({ }^{3}\right)\right)\right)$, and thus, we get the spectrum space ${ }^{3}$. This simple example will make us propose the $(M)$ later.

In Leibniz-Clarke correspondence (1715-1716), they (i.e., Leibniz and Clarke (=Newton's friend)) discussed "space-time problem". Their ideas are summarized as follows:

$$
(L)\left\{\begin{array}{l}
{ }^{\circledR}: \text { realistic "space-time" } \leftarrow \text { Newton,Clarke } \\
\text { L: linguistic "space-time" } \leftarrow \text { Leibniz }
\end{array}\right.
$$

That is, Newton considered "What is space-time?". On the other hand, Leibniz considered "How should the term: space-time be used?" Measurement theory is in Leibniz's side, and asserts that

(M): Space should be described as a kind of spectrum space. And time should be described as a kind of tree. In other words, time is represented by a parameter $t$ in a linear orderedtree $T$.

Therefore, we think that the Leibniz-Clarke debates should be essentially regarded as "the linguistic world

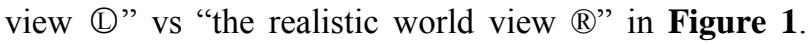
The above $(M)$ should be added to Interpretation $(E)$ as sub-interpretation of measurement theory.

\subsection{Observer's Time}

It is usual to consider that quantum mechanics and observer's time are incompatible. This leads Interpretation $\left(E_{3}\right)$, which says that observer's time is nonsense in measurement theory. That is, there is no tense - past, present, future - in measurement theory, and therefore, in science. Many philosophers (e.g., Augustinus, Bergson, Heidegger, etc.) tried to understand observer's time. However, from the scientific point of view, their attempts may be reckless. From the measurement theoretical point of view, we feel sympathy for J. McTaggart, whose paradox [14] suggests that observer's time leads science to inconsistency.

\subsection{Linguistic Turn (Figure 1: (3), (5))}

For the question "Which came first, the world or the language?", two answers (the realistic world view and the linguistic world view) are possible. However, as mentioned in the $(F)$, measurement theory is in the side of "the language came first" (due to Saussure, Wittgenstein, etc.).

Note that two kinds of linguistic turns (i.e., (3) and (5)) are mentioned in Figure 1. Here, the (3) is the turn from physics to language, and the (5) is the turn from "brain" to language. Also, note that the linguistic world view $(F)$ is essentially the same as the following Wittgenstein's famous statement:

$(N)$ The limits of my language mean the limits of my world.

The interpretation $\left(E_{3}\right)$ says that the statement concerning Schrödinger's cat cannot be described in measurement theory. Therefore, in the sense of the above $(N)$, Schrödinger's cat (and moreover, Einstein's world, i.e., the theory of relativity) is out of the world described by measurement theory. This means that Schrödinger's cat is not paradoxical in the framework of measurement theory. This is the advantage of our linguistic approach to quantum mechanics. Again, see our assertion $(F)$.

Also, the assertion $(C)$ is similar to Kant's main assertion ("synthetic a priori judgment") in his famous book "Critique of Pure Reason [15]", that is, the two are similar in the sense of no experimental validation. Therefore, we want to consider the following correspondence:

$(O)$ [synthetic a priori judgment] $\leftrightarrow$ [Axioms 1 and 2] However, as mentioned in $\left(J_{3}\right)$, Kant's epistemological approach (i.e., Copernican turn) is rather psychological and not linguistic, in spite that his purpose is philosophy (i.e., the world view) and not psychology (or brain science).

\subsection{Parmenides Philosophy}

About 2500 years ago, Parmenides said that

$(P)$ There is no "plurality", but only "one". And therefore, there is no movement.

We want to regard this $(P)$ as the origin of Interpretation $\left(E_{2}\right)$ (i.e., "Only one measurement is permitted. And therefore, a state never moves."). The $\left(E_{2}\right)$, the superiority of the Heisenberg picture to the Schrödinger picture, is the most technical and mathematical interpretation in the $(E)$. And, it is frequently used in the applications of measurement theory ( $c f$. [1-6]). And hence, it is recommended to practice how to use $\left(E_{2}\right)$ in [1-6]. At least, see Example 4 in [1].

\subsection{Zeno's Paradoxes}

Zeno, the student of Parmenides, proposed several paradoxes concerning movement. The following "Achilles and the tortoise" is most famous.

[Achilles and the tortoise] In a race, the quickest runner can never overtake the slowest, since the pursuer must first reach the point whence the pursued started, so that the slower must always hold a lead.

Beginners of philosophy may have a question:

(Q) Why have philosophers investigated such an easy problem during 2500 years?

However, it should be noted that "Achilles and the tortoise" is not an elementary mathematical problem concerning geometric series. Since Parmenides and Zeno were philosophers, it is natural to consider that Zeno's paradoxes should be regarded as the problem concerning 
world view. That is, we believe that Zeno's question is as follows:

(R) In what kind of world view (in Figure 1) should

Zeno's paradoxes be understood? And further, if the proper world view is not in Figure 1, propose the new world view in which Zeno's paradoxes can be discussed!

It is clear that Zeno's paradoxes are not in physics, and thus, Newtonian mechanics, quantum mechanics and the theory of relativity and so on are not proper for the answer to the problem $(R)$. We assert that classical measurement theory is the proper world view, in which Zeno's paradoxes are described. The classical measurement theoretical description of Zeno's paradoxes is easy. In fact, it was simply shown in [6].

Readers may be interested in the unnatural situation such that "Achilles" and "tortoise" are quantum particles (i.e., Zeno's paradoxes in quantum mechanics). Although we have no clear answer to this problem, our paper [16] may be helpful. Also, for the more unnatural situation (i.e., Zeno's paradoxes in the theory of relativity), see [17].

It is interesting and strange to see that we already have the world description methods (i.e., Newtonian mechanics, quantum mechanics and the theory of relativity) for the unnatural situations, but, we have no world description method for the natural situation if we do not know measurement theory.

\subsection{Syllogism}

As an example of syllogism, the following example (due to Aristotle) is famous.

(S) Since Socrates is a man and all men are mortal, it follows that Socrates is mortal.

However, it should be noted that there is a great gap between the $(S)$ and the following mathematical syllogism: (T) $A \Rightarrow B, B \Rightarrow C$, then, it follows that $A \Rightarrow C$.

That is because the $(T)$ is merely mathematical rule and not the world view, and therefore, it is not guaranteed that the rule $(T)$ is applicable to the world $(S)$. In fact, as mentioned in the following section, the $(S)$ does not hold in quantum cases.

Now, we are in the same situation such as $(R)$. That is, we have a similar question (i.e., $\left.(R) \approx\left(R^{\prime}\right)\right)$ :

$\left(R^{\prime}\right)$ In what kind of world view (in Figure 1) should the phenomenon $(S)$ be described? And further, if the proper world view is not in Figure 1, propose the new world view!

We assert that classical measurement theory is the proper world view. In fact, in [2], the above phenomenon $(S)$ is described as a theorem in classical measurement theory.

\subsection{Syllogism Does Not Hold in Quantum Systems}

Since we understand that EPR-paradox [10] [resp. Bell's inequality [18]] suggests that quantum mechanics and syllogism [resp. locality] are incompatible, we are not going to assert our originality of the following example. However, in order to promote the understanding of the gap between $(S)$ and $(T)$, let us add a simple and clear example as follows.

Consider the three dimensional Hilbert space $H={ }^{3}$, And therefore, we get the non-commutative $C^{*}$-algebra $A=B(H)$, that is, the algebra composed of all $3 \times 3$ matrices. Define the three sets such as

$$
A=\left\{a_{1}, a_{2}\right\}, \quad B=\left\{b_{1}, b_{2}\right\}, \quad C=\left\{c_{1}, c_{2}\right\}
$$

Here, define the observable $O_{A}:=\left(A, 2^{A}, F_{A}\right)$ in $B(H)$ such that

$$
F_{A}\left(\left\{a_{1}\right\}\right)=\left[\begin{array}{lll}
1 & 0 & 0 \\
0 & 0 & 0 \\
0 & 0 & 0
\end{array}\right], \quad F_{A}\left(\left\{a_{2}\right\}\right)=\left[\begin{array}{lll}
0 & 0 & 0 \\
0 & 1 & 0 \\
0 & 0 & 1
\end{array}\right] .
$$

And define $O_{B}:=\left(B, 2^{B}, F_{B}\right)$ such that

$$
F_{B}\left(\left\{b_{1}\right\}\right)=\left[\begin{array}{lll}
1 & 0 & 0 \\
0 & 1 & 0 \\
0 & 0 & 0
\end{array}\right], \quad F_{B}\left(\left\{b_{2}\right\}\right)=\left[\begin{array}{lll}
0 & 0 & 0 \\
0 & 0 & 0 \\
0 & 0 & 1
\end{array}\right] .
$$

And further, define $O_{C}:=\left(C, 2^{C}, F_{C}\right)$ such that

$$
\begin{aligned}
F_{C}\left(\left\{c_{1}\right\}\right) & =\left[\begin{array}{ccc}
1 / 2 & 1 / 2 & 0 \\
1 / 2 & 1 / 2 & 0 \\
0 & 0 & 0
\end{array}\right], \\
F_{C}\left(\left\{c_{2}\right\}\right) & =\left[\begin{array}{ccc}
1 / 2 & -1 / 2 & 0 \\
-1 / 2 & 1 / 2 & 0 \\
0 & 0 & 1
\end{array}\right] .
\end{aligned}
$$

Define the state $\rho_{0}$ such that

$$
\rho_{0}=\left\lfloor\left[\begin{array}{l}
\alpha \\
\alpha \\
\beta
\end{array}\right]\right\rangle /\left[\begin{array}{l}
\alpha \\
\alpha \\
\beta
\end{array}\right] \mid\left(\alpha, \beta \in, 2|\alpha|^{2}+|\beta|^{2}=1\right) .
$$

Since $O_{A}$ and $O_{B}$ [resp. $O_{B}$ and $O_{C}$ ] commute, we get the product observable $O_{A B}=\left(A \times B, 2^{A \times B}, F_{A B}\right)$, where

$$
F_{A B}(\{(a, b)\})=F_{A}(\{a\}) F_{B}(\{b\}) \quad(\forall(a, b) \in A \times B)
$$

Similarly, we get the product observable

$O_{B C}=\left(B \times C, 2^{B \times C}, F_{B C}\right)$.

Here, we see the following $\left(U_{1}\right)$ and $\left(U_{2}\right)$ :

$\left(U_{1}\right)$ Let $(x, y) \in\left\{a_{1}, a_{2}\right\} \times\left\{b_{1}, b_{2}\right\}$ be a measured value obtained by the measurement $M_{B(H)}\left(O_{A B}, S_{\left[\rho_{0}\right]}\right)$. Then we see:

$$
" x=a_{1} " \Rightarrow " y=b_{1} "
$$


since

$$
\begin{aligned}
& \rho_{0}\left(F_{A B}\left(\left\{\left(a_{1}, b_{2}\right)\right\}\right)\right) \\
& =\left\langle\left[\begin{array}{l}
\alpha \\
\alpha \\
\beta
\end{array}\right],\left[\begin{array}{lll}
1 & 0 & 0 \\
0 & 0 & 0 \\
0 & 0 & 0
\end{array}\right] \cdot\left[\begin{array}{lll}
0 & 0 & 0 \\
0 & 0 & 0 \\
0 & 0 & 1
\end{array}\right]\left[\begin{array}{l}
\alpha \\
\alpha \\
\beta
\end{array}\right]\right\rangle_{3}=0
\end{aligned}
$$

$\left(U_{2}\right)$ Let $(y, z) \in\left\{b_{1}, b_{2}\right\} \times\left\{c_{1}, c_{2}\right\}$ be a measured value obtained by the measurement $M_{B(H)}\left(O_{B C}, S_{\left[\rho_{0}\right]}\right)$. Then we see:

$$
" y=b_{1} " \Rightarrow " z=c_{1} "
$$

since

$$
\begin{aligned}
& \rho_{0}\left(F_{B C}\left(\left\{\left(b_{1}, c_{2}\right)\right\}\right)\right) \\
& =\left\langle\left[\begin{array}{l}
\alpha \\
\alpha \\
\beta
\end{array}\right],\left[\begin{array}{lll}
1 & 0 & 0 \\
0 & 1 & 0 \\
0 & 0 & 0
\end{array}\right] \cdot\left[\begin{array}{ccc}
1 / 2 & -1 / 2 & 0 \\
-1 / 2 & 1 / 2 & 0 \\
0 & 0 & 1
\end{array}\right]\left[\begin{array}{l}
\alpha \\
\alpha \\
\beta
\end{array}\right]\right\rangle_{3} \\
& =0
\end{aligned}
$$

Thus, some may, from the (4) and (5), conclude that

$$
\text { " } x=a_{1} " \Rightarrow " z=c_{1} " \text {. }
$$

However, this (6) is wrong. Recall Interpretation $\left(E_{2}\right)$, i.e., "only one measurement is permitted". Thus, we must construct the observable

$O_{A B C}=\left(A \times B \times C, 2^{A \times B \times C}, F_{A B C}\right)$, where

$$
\left(F_{A B C}\right)(\{(a, b)\} \times C)=F_{A B}(\{(a, b)\}) \quad(\forall(a, b) \in A \times B)
$$

and

$$
\left(F_{A B C}\right)(A \times\{(b, c)\})=F_{B C}(\{(b, c)\}) \quad(\forall(b, c) \in B \times C)
$$

And further, we have to take the measurement $M_{B(H)}\left(O_{A B C}, S_{\left[\rho_{0}\right]}\right)$. However, it is easily shown that such observable $O_{A B C}=\left(A \times B \times C, 2^{A \times B \times C}, F_{A B C}\right)$ does not exist. Hence, we can conclude that syllogism does not generally hold in quantum measurement theory. We believe that the above argument is essentially the same as that of EPR-paradox [10]. Also, for the precise definition of "implication $\Rightarrow$ " in measurement theory, see [2].

\section{Conclusions}

As mentioned in [1-6], measurement theory (including several conventional system theories, e.g., statistics, dynamical system theory, quantum system theory, etc.) is one of the most useful theories in science. Following the well-known proverb: "A sound mind in a sound body", we consider: "A good philosophy in a very useful theory." For example, "A good realistic philosophy in the theory of relativity" is clearly sure. Therefore, we believe that a good philosophy has to be hidden behind measurement theory. This belief makes us write this paper. And, we want to conclude "A good linguistic philosophy in quantum mechanics (or MT)".

In the abstract of this paper, we promised readers to show a good linguistic philosophy in quantum mechanics. Now we are convinced that this promise is kept. That is because we believe that readers look at the moment philosophy changes to science (Figure 1: (8)).

Dr. Hawking said in his best seller book [19]: Philosophers reduced the scope of their inquiries so much that Wittgenstein the most famous philosopher this century, said "The sole remaining task for philosophy is the analysis of language." What a comedown from the great tradition of philosophy from Aristotle to Kant! We think that this is not only his opinion but also most scientists' opinion. And moreover, we mostly agree with him. However, we believe that it is worth reconsidering the series (D) (i.e., the linguistic world view) in Figure 1.

In this paper, we only studied a few about the relation between quantum mechanics and philosophy. Thus, we hope that our proposal will be discussed and examined from various viewpoints.

\section{REFERENCES}

[1] S. Ishikawa, "A New Interpretation of Quantum Mechanics," Journal of quantum information science, Vol. 1, No. 2, 2011, pp. 35-42.

[2] S. Ishikawa, "Fuzzy Inferences by Algebraic Method," Fuzzy Sets and Systems, Vol. 87, No. 2, 1997, pp. 181200. doi:10.1016/S0165-0114(96)00035-8

[3] S. Ishikawa, "A Quantum Mechanical Approach to Fuzzy Theory," Fuzzy Sets and Systems, Vol. 90, No. 3, 1997, pp. 277-306. doi:10.1016/S0165-0114(96)00114-5

[4] S. Ishikawa, "Statistics in Measurements," Fuzzy Sets and Systems, Vol. 116, No. 6, 2000, pp. 141-154. doi:10.1016/S0165-0114(98)00280-2

[5] S. Ishikawa, "Mathematical Foundations of Measurement Theory," Keio University Press Inc., Yokohama, 2006, pp. 1-335. http://www.keio-up.co.jp/kup/mfomt/

[6] S. Ishikawa, "Dynamical Systems, Measurements, Quantitative Language and Zeno's Paradoxes," Far East Journal of Dynamical Systems, Vol. 10, No. 3, 2008, pp. 277-292.

[7] G. J. Murphy, " $C^{*}$-Algebras and Operator Theory," Academic Press, London, 1990.

[8] J. von Neumann, "Mathematical Foundations of Quantum Mechanics," Springer Verlag, Berlin, 1932.

[9] E. B. Davies, "Quantum Theory of Open Systems," Academic Press, London, 1976

[10] A. Einstein, B. Podolosky and N. Rosen, "Can Quantum-Mechanical Description of Physical Reality Be Considered Complete?" Physical Review, Vol. 47, No. 10, 1935, pp. 777-780. doi:10.1103/PhysRev.47.777 
[11] N. Bohr, "Can Quantum-Mechanical Description of Physical Reality Be Considered Complete?" Physical Review, Vol. 47, No. 8, 1935, pp. 696-702. doi:10.1103/PhysRev.48.696

[12] K. R. Popper, "The Logic of Scientific Discovery," Basic Books, Inc., New York, 1959.

[13] A. Kolmogorov, "Foundations of the Theory of Probability (Translation)," 2nd Edition, Chelsea Publishing Co., New York, 1960.

[14] J. M. E. McTaggart, "The Unreality of Time," Mind (A Quarterly Review of Psychology and Philosophy), Vol. 17, 1908, pp. 457-474. doi:10.1093/mind/XVII.4.457

[15] I. Kant, "Critique of Pure Reason," In: P. Guyer and A. W. Wood, Eds., Cambridge University Press, Cambridge, 1999.

[16] S. Ishikawa, T. Arai and T. Kawai, "Numerical Analysis of Trajectories of a Quantum Particle in Two-Slit Ex- periment," International Journal of Theoretical Physics, Vol. 33, No. 6, 1993, pp. 1265-1274. doi:10.1007/BF00670793

[17] J. Mazur, "Motion Paradox, The 2500-Year-Old Puzzle Behind All the Mysteries of Time and Space," Dutton Adult, Boston, 2007.

[18] J. S. Bell, "On the Einstein-Podolosky-Rosen Paradox," Physics, Vol. 1, No. 4, 1966, pp. 195-200.

[19] S. Hawking, "A Brief History of Time: From the Big Bang to Black Holes," Bantam, New York, 1990.

[20] S. Ishikawa, "A Measurement Theoretical Foundation of Statistics," Applied Mathematics, Vol. 3, No. 3, 2012, pp. 283-292.

[21] S. Ishikawa, "Ergodic Hypothesis and Equilibrium Statistical Mechanics in the Quantum Mechanical World View," World Journal of Mechanics, Vol. 2, No. 2, 2012, in Press. 